\title{
Light Activated Recombination
}

\author{
Kristian H. Link, Youheng Shi, and John T. Koh \\ Department of Chemistry and Biochemistry, University of Delaware, Newark, Delaware, 19716, \\ johnkoh@udel.edu
}

\begin{abstract}
Methods to remotely regulate gene expression have revolutionized the study of gene function by enabling one to selectively turn on or turn off genes in a temporally defined manner.

However, many genes such as morphogens or neuronal guidance cues, can only properly elicit their actions through their precise spatial and temporal patterning in tissues. Several groups have used various strategies to photocaged biopolymers, (DNA, RNA or proteins) as a way to control gene expression in a spatiotemporally defined manner. ${ }^{1-3}$ Unfortunately, application of these methods is often limited by the difficulties associated with introducing these caged biopolymers into multi-cellular systems. A few years ago we introduced one of the first methods to control gene expression by using photo-caged small-molecule agonist of nuclear hormone receptors (NHRs) that can freely diffuse into cultured cells. ${ }^{4}$ Photo-caged agonists of NHRs can be used to control the expression of genes in a spatially discrete manner in multicellular systems. ${ }^{5}$ However, the duration of transcription response is limited by the diffusion of the uncaged ligand out of the cell, and the stability of the ligand-receptor complex. ${ }^{6}$ Herein we describe a general method to permanently switch on or off gene expression by controlling recombination in a light-directed manner with the ligand dependent recombinase Cre-ER ${ }^{\mathrm{T}}$, a fusion of the site-specific recombinase Cre and a tamoxifen selective mutant of the ligand binding domain of the estrogen receptor (ER) first developed by Chambon. ${ }^{7}$
\end{abstract}

Cre recombinase catalyzes the homologous recombination of DNA strands at loxP recognition sequences. Cre can serve to permanently turn-on (or turn-off) genes that are flanked by the loxP sites such that recombination places the gene of interest directly downstream of a promotor (Figure 1c). Recombination permanently alters gene expression for a cell and its progeny. Cre$\mathrm{ER}^{\mathrm{T}}$ can be used to conditionally control Cre activity using the ER antagonist tamoxifen (Tam), which dissociates the unliganded ER ligand-binding domain from heat-shock proteins.

We evaluated the ability of the Nitroveratryl (Nv) protected form of 4-hydroxytamoxifen (NvHTam), ${ }^{8}$ to control recombination in an exposure-dependent manner in HEK293 cells stably expressing a zeomycin selectable $\mathrm{pCre}-\mathrm{ER}^{\mathrm{T}} / \mathrm{Zeo}$ and the recombination reporter $\mathrm{pLacZ}(\mathrm{LNL})$ (Figure 1a and 1c). ${ }^{7}$ Cells grown in media containing Nv-HTam show exposure-dependent expression of $\beta$-galactosidase (Figure $2 \mathrm{c}$ and $2 \mathrm{~d}$ ). After a $67 \mathrm{~h}$ incubation, cells treated with Nv-HTam and kept in the dark show no significant reporter gene expression, whereas cells briefly irradiated with UV light (>345 nm) show significant $\beta$-galactosidase expression as evident from x-gal staining.

Spatial patterning of gene expression in tissues, requires that the ligand be effective when released within only a subpopulation of cells. In this case the intracellular concentration of ligand will quickly decrease as the free ligand diffuses out of the cell and is diluted in the surrounding media. When cells are exposed to only intracellularly released HTam, achieved by exchanging the media immediately after irradiation, no significant recombination is observed (see below). Therefore, although Nv-HTam is a photocaged trigger of Cre-ER ${ }^{\mathrm{T}}$, its 
potential applications as a tool for photo-patterning are limited by the short lifetime of the HTam/Cre-ER ${ }^{\mathrm{T}}$ complex compared to the $72 \mathrm{~h}$ incubation required to achieve efficient recombination.

The lifetime of a ligand-NHR complex is typically controlled by the ligand's off-rate and the rate of receptor turn over by proteolysis. ${ }^{6}$ Tamoxifen aziridine, (Taz) is an ER antagonist that forms a covalent adduct with Cys530 of ER (Figure 1b). Although the ER/estradiol complex is rapidly turned over through the ubiquitin-proteasome pathway, the ER/Taz complex has a remarkably long half-life $\left(\mathrm{t}_{1 / 2} \approx 32 \mathrm{~h}\right) .9,10$ This suggests that the Taz/Cre-ER ${ }^{\mathrm{T}}$ complex may have a long enough lifetime to mediate recombination from one or just a few intracellular exposures. We therefore synthesized a photocaged analog of 4-hydroxytamoxifen aziridine, Nv-HTaz, and evaluated its ability to control recombination in cells expressing Cre-ER ${ }^{\mathrm{T}}$ and the recombination reporter (Figure 1b).

$\mathrm{Nv}-\mathrm{HTaz}$ is also a photocaged trigger for $\mathrm{CreER}^{\mathrm{T}}$ that only activates recombination upon exposure (Figure 3a \& 3b). Similar to Tam, high levels of recombination are observed when cells are left in contact with photo-released ligand for the entire $67 \mathrm{~h}$ incubation. When exposed to only intracellularly released ligand, the covalent binding Nv-HTaz provides a distinct advantage over Nv-HTam, providing significant recombination from a single exposure (Figure $3 \mathrm{c} \& 3 \mathrm{~d}$ ), although, the fraction of recombined cells is clearly less than is observed when cells are treated continuously with Tam for 72 hours.

Only a modest fraction of the cells undergo recombination after a single exposure to uncage intracellular Nv-HTaz, however, the fraction of recombined cells can be significantly increased by using just three exposures at $24 \mathrm{~h}$ intervals (Figure $3 \mathrm{e} \& 3 \mathrm{f}$ ). As not every cell in this late passage cell line is able to undergo recombination when continuously treated with Tam, we quantified the inducible $\beta$-gal activity by o-nitrophenygalactosidase (ONPG) assay (Figure 4). Using three exposures $26 \%$ of the possible $\beta$-gal activity could be obtained with $500 \mathrm{nM}$ of intracellular Nv-Htaz. Under identical conditions, Nv-HTam or $250 \mathrm{nM}$ NvTaz does not activate expression significantly greater than background.

The covalent-modifying Nv-HTaz used in combination with Cre-ER ${ }^{\mathrm{T}}$ is a powerful tool for irreversibly controlling recombination in a spatially and temporally directed manner. The NvHTaz/Cre-ER ${ }^{\mathrm{T}}$ system represents perhaps the first example of spatio-temporal control of recombination through a photo-inducible system.

\section{Supplementary Material}

Refer to Web version on PubMed Central for supplementary material.

\section{Acknowledgements}

We thank Robert Nardone, Prof. M. C. Farach-Carson and Pam Trowbridge, for helpful discussions. Dr. Steven Bai for NMR assistance. Vectors $\mathrm{pCre}^{-\mathrm{ER}^{\mathrm{T}}}$ and $\mathrm{pLacZ}(\mathrm{LNL})$ were a generous gift from Prof. Pierre. Chambon. We thank NIH DK054257 and NS49523 for financial support.

\section{References}

1. Haselton FR, Tseng WC, Chang MS. Invest Ophthalmol Vis Sci 1997;38:2082-2082.

2. Ando H, Furuta T, Tsien RY, Okamoto H. Nature Genet 2001;28:317-325. [PubMed: 11479592]

3. Cambridge SB, Davis RL, Minden JS. Science 1997;277:825-828. [PubMed: 9242613]

4. Cruz FG, Koh JT, Link KH. J Am Chem Soc 2000;122:8777-8778.

5. Lin WY, Albanese C, Pestell RG, Lawrence DS. Chem Biol 2002;9:1347-1353. [PubMed: 12498888]

6. Link KH, Cruz FG, Ye HF, O'Reilly K, Dowdell S, Koh JT. Bioorg Med Chem 2004;12:5949-5959. [PubMed: 15498671] 
7. Feil R, Brocard J, Mascrez B, LeMeur M, Metzger D, Chambon P. Proc Natl Acad Sci USA 1996;93:10887-10890. [PubMed: 8855277]

8. Shi YH, Koh JT. Chembiochem 2004;5:788-796. [PubMed: 15174161]

9. Katzenellenbogen JA, Carlson KE, Heiman DF, Robertson DW, Wei LL, Katzenellenbogen BS. J Biol Chem 1983;258:3487-3495. [PubMed: 6833211]

10. Wijayaratne AL, McDonnell DP. J Biol Chem 2001;276:35684-35692. [PubMed: 11473106] 
a.

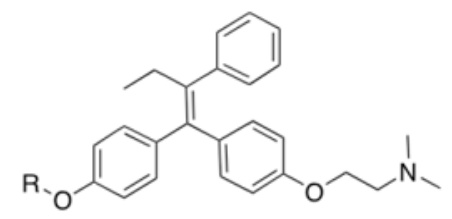

HTam: $\mathrm{R}=\mathrm{H}$

Nv-HTam: R = nitroveratryl, (Nv)

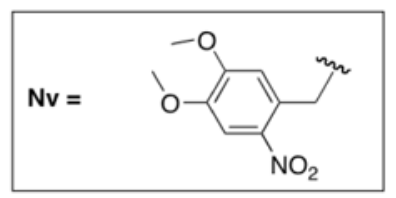

c.
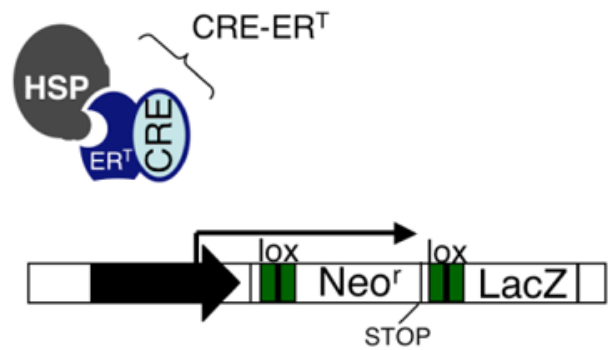

b.

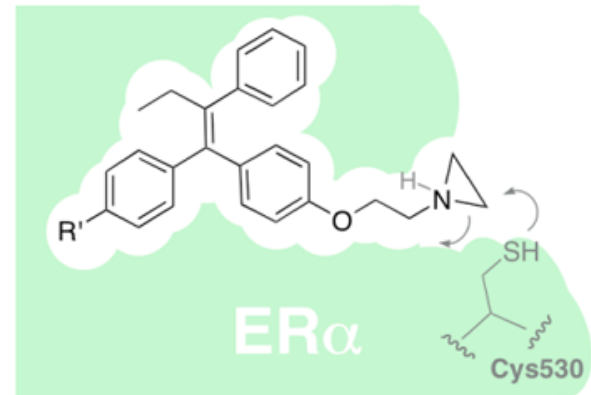

Taz; $\mathrm{R}^{\prime}=\mathrm{H}$

HTaz; $\mathrm{R}^{\prime}=\mathrm{OH}$

$\mathrm{Nv}-\mathrm{HTaz}=\mathrm{R}^{\prime}=\mathrm{NvO}$

\section{LoxP: $\square=$ 5'-TATAACTTCGTATAGATATGCTATACGAAGTTAT-3'}

FIGURE 1.

(A). Photocaged hydroxytamoxifen (HTam). (B). Taz forms a covalent adduct with Cys 530 of ER; Nv-HTaz is a photocaged Taz analog. (C). Cre-ER ${ }^{\mathrm{T}}$ can be used to permanently turn on reporter gene (LacZ) expression (and turn off $\mathrm{Neo}^{\mathrm{r}}$ ) through recombination. (HSP; heat shock protein, $\mathrm{Neo}^{\mathrm{r}}$; neomycin resistance). 

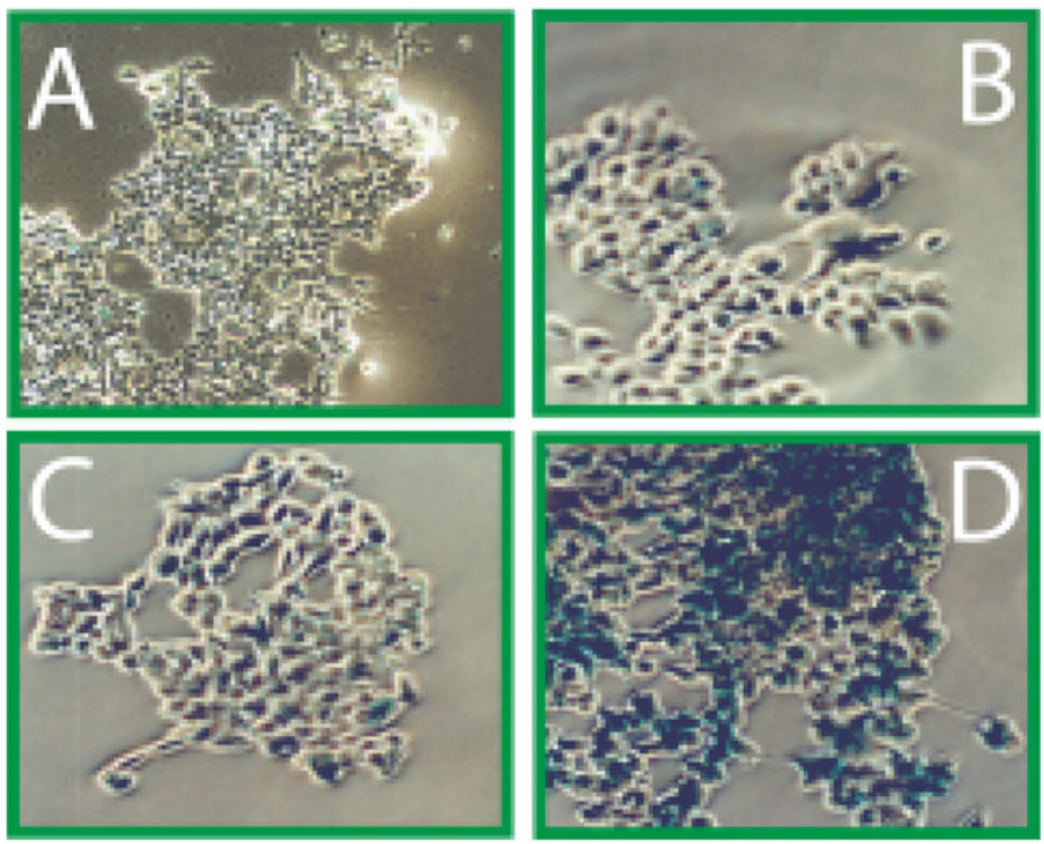

FIGURE 2.

$\mathrm{Nv}$-HTam can regulate recombination dependent $\beta$-gal expression (x-gal stain) in HEK293 cells expressing pLacZ(LNL) and pCre-ER ${ }^{\mathrm{T}} / \mathrm{Zeo}$. (A) No ligand, unexposed. (B) No ligand, exposed (180s $\times 3 \mathrm{~W})$. (c) Nv-HTam, unexposed. (D) Nv-HTam, exposed. Intracellular and extacellular ligand $(200 \mathrm{nM})$ with $200 \mathrm{nM}$ trans-retinoic acid (tRA). 

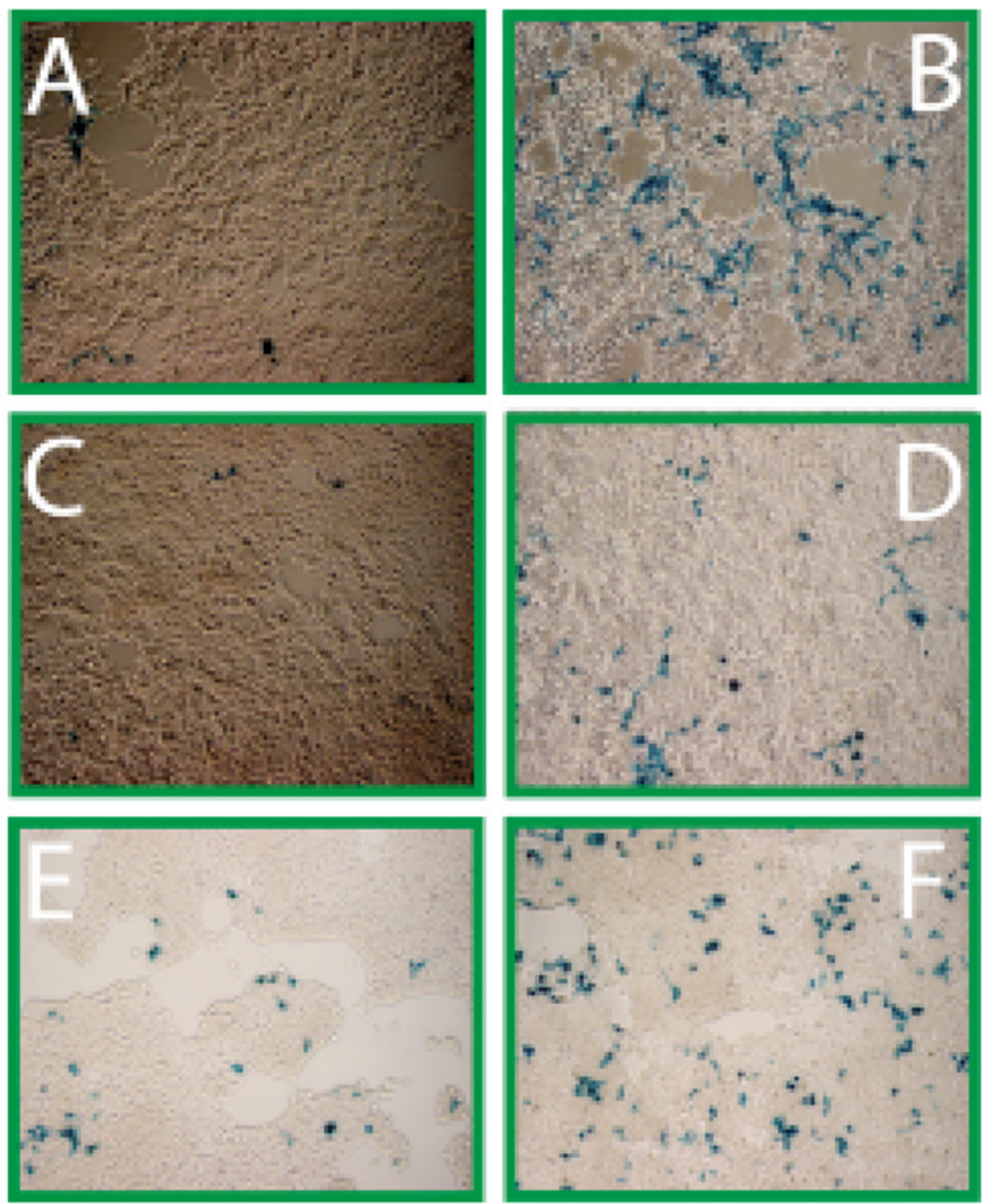

FIGURE 3.

Comparison of Nv-HTam and Nv-HTaz mediated recombination after $72 \mathrm{~h}$ (x-gal staining). (A) Nv-HTaz, unexposed. (B) Intra- and extra-cellular uncaged Nv-HTaz, exposed (180s) (C) Intracellularly uncaged Nv-HTam, exposed (180s). (D) Intracellularly uncaged Nv-HTaz, exposed (180s). (E) Intracellularly uncaged Nv-HTam, exposed 3-times (45s). (F) Intracellularly uncaged Nv-HTaz, exposed 3 times (45s). $1000 \mathrm{nM}$ ligand plus of $200 \mathrm{nM}$ tRA. 


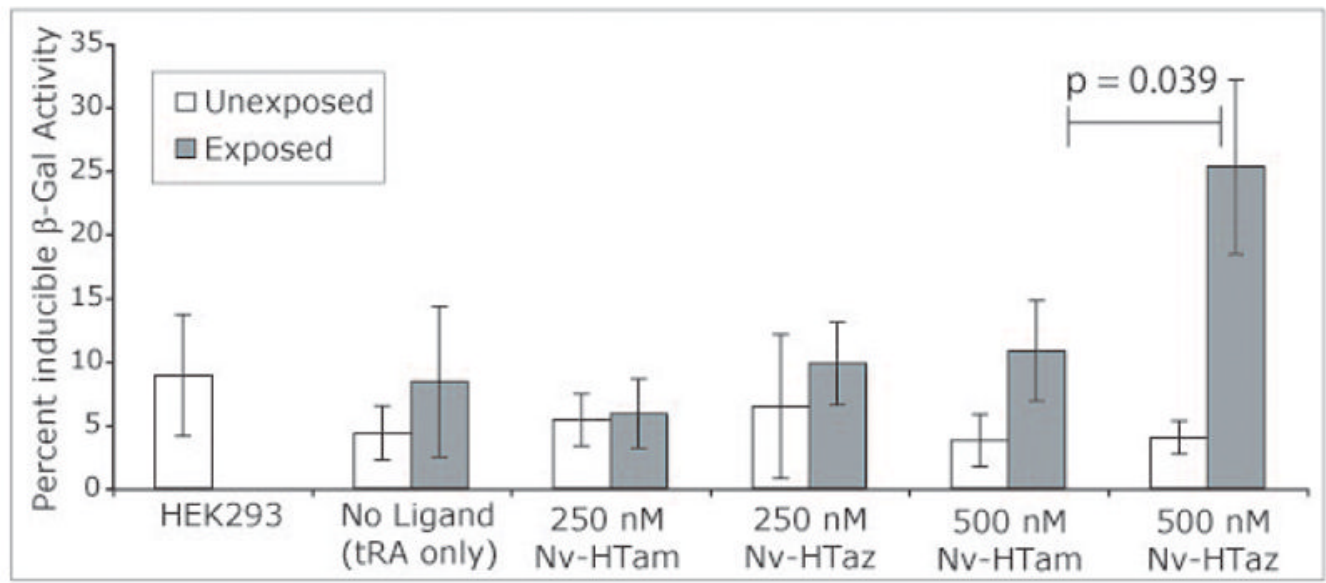

FIGURE 4.

$\beta$-gal expression measured by OPNG assay for cells treated with Nv-HTaz or Nv-HTam with $200 \mathrm{nM}$ tRA and exposed three times at $24 \mathrm{~h}$ intervals $(45 \mathrm{~s} \times 3 \mathrm{~W}) .(\mathrm{p}$; unpaired $\mathrm{t}$-test) 